International Journal of Pure and Applied Mathematics

Volume 85 No. 1 2013, 23-31

ISSN: 1311-8080 (printed version); ISSN: 1314-3395 (on-line version)

url: http://www.ijpam.eu

doi: http://dx.doi.org/10.12732/ijpam.v85i1.3

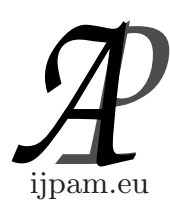

\title{
THREE STEP ITERATION SCHEME FOR M-CONTRACTIVE CONDITION IN BANACH SPACE
}

\author{
M.R. Yadav ${ }^{1}$, B.S. Thakur², Ajay Kumar Sharma ${ }^{3}$ \\ ${ }^{1,2}$ School of Studies in Mathematics \\ Pt. Ravishankar Shukla University \\ Raipur, Chhattisgarh, 492010, INDIA \\ ${ }^{3}$ Seth Phoolchand College \\ Navapara Rajim, Dist. Raipur, Chhattisgarh, INDIA
}

\begin{abstract}
Our aim in this paper is to prove a strong convergence theorem by three-step iteration scheme with respect to contractive condition in a Banach space. Our results obtained in this paper is new extension as well as refinement of previous known results.
\end{abstract}

AMS Subject Classification: 47H05, 47H10, 49M05

Key Words: three-step iteration scheme, contractive condition, Banach space, strong convergence, common fixed point

\section{Introduction}

Let $X$ be a Banach space, $K$ be a nonempty closed convex subset of $X$ and $T: K \rightarrow K$ be a self mapping of $K$. Throughout this paper, $\mathbb{N}$ denotes the set of all positive integers and $F(T) \neq \phi$ i.e., $F(T)=\{x \in K: T x=x\}$. Suppose $\left\{\alpha_{n}\right\}$ and $\left\{\beta_{n}\right\}$ are two sequences in real number $[0,1]$.

Received: August 27, 2012

(C) 2013 Academic Publications, Ltd. url: www.acadpubl.eu

${ }^{\S}$ Correspondence author 
The Picard and Mann [7] iteration schemes for a mapping $T: K \rightarrow K$ are defined by

$$
\left\{\begin{array}{l}
x_{1}=x_{0} \in K \\
x_{n+1}=T x_{n}
\end{array}\right.
$$

and

$$
\left\{\begin{array}{l}
x_{1}=x_{0} \in K \\
x_{n+1}=\left(1-\alpha_{n}\right) x_{n}+\alpha_{n} T x_{n}, n \in \mathbb{N}
\end{array}\right.
$$

where $\left\{\alpha_{n}\right\}$ is in $(0,1)$.

The sequence $\left\{x_{n}\right\}_{n=0}^{\infty}$ defined by

$$
\left\{\begin{array}{l}
x_{1}=x_{0} \in K \\
x_{n+1}=\left(1-\alpha_{n}\right) x_{n}+\alpha_{n} T y_{n}, \\
y_{n}=\left(1-\beta_{n}\right) x_{n}+\beta_{n} T x_{n}, n \in \mathbb{N}
\end{array}\right.
$$

is known as the Ishikawa iteration process [5].

In this paper, we define a three-step iteration scheme as follows:

Let $K$ be a nonempty, closed and convex subset of a Banach space $X$. Suppose $T: K \rightarrow K$ be two nonlinear operators and $\left\{x_{n}\right\}_{n=0}^{\infty}$ be the sequence in $[0,1]$.

$$
\left\{\begin{array}{l}
x_{1}=x_{0} \in K \\
x_{n+1}=\left(1-\alpha_{n}\right) z_{n}+\alpha_{n} T z_{n} \\
y_{n}=\left(1-\beta_{n}\right) x_{n}+\beta_{n} T x_{n} \\
z_{n}=\left(1-\gamma_{n}\right) y_{n}+\gamma_{n} T y_{n}, n \in \mathbb{N}
\end{array}\right.
$$

where $\left\{\alpha_{n}\right\},\left\{\beta_{n}\right\}$ and $\left\{\gamma_{n}\right\}$ are sequences in $[0,1]$ with $\alpha_{n}+\beta_{n}+\gamma_{n}=1$.

We note that, when $\beta_{n}=0$, then the iteration scheme (4) reduce to the iteration given by Thianwan [11],

$$
\left\{\begin{array}{l}
x_{1}=x_{0} \in K \\
x_{n+1}=\left(1-\alpha_{n}\right) y_{n}+\alpha_{n} T y_{n} \\
y_{n}=\left(1-\beta_{n}\right) x_{n}+\beta_{n} T x_{n}, n \in \mathbb{N}
\end{array}\right.
$$

where $\left\{\alpha_{n}\right\}$ and $\left\{\beta_{n}\right\}$ are sequences in $[0,1]$.

When $\beta_{n}=0$ the iteration (5) reduces to the Mann iteration (2).

The following theorem was obtained by the Zamfirescu [12]. 
Theorem 1. Let $(X, d)$ be complete metric space and $T: X \rightarrow X$ a map for which there exist the real number $a, b$, and $c$ satisfying $0<a<1$, $b, c \in(0,1 / 2)$ such that for each pair $x, y$ in $X$, at least one the following is true: $\left(z_{1}\right) d(T x, T y) \leq a d(x, y)$ $\left(z_{2}\right) d(T x, T y) \leq b[d(x, T x)+d(y, T y)]$, $\left(z_{3}\right) d(T x, T y) \leq c[d(x, T y)+d(y, T x)]$. Then $T$ is a Picard operator $x_{n+1}=T x_{n}$.

An operator $T$ satisfying the contractive condition $\left(z_{1}\right),\left(z_{2}\right)$ and $\left(z_{3}\right)$ in Theorem 1, above, is called a Zamfirescu operator.

\section{Preliminaries}

In recent years, Mann and Ishikawa iteration schemes have been studied extensively by many authors to solve one parameter nonlinear operator equations as well as variational inequalities in Hilbert space and Banach spaces. Many authors study Rhoades ([9], [10]) emplyed the Zamfirescu condition to prove several convergence results for Mann and Ishikawa iteration process in a uniformly convex Banach space. The results of Rhoades ([9], [10]) were also extended by Berinde [3] to an arbitrary Banach space for the same fixed point iteration processes. Recently, many research papers have been published on the iterative approximation of fixed points for contractive type, quasi-contractive and Zamfirescu operators using several iteration schemes, for example see ([3], [4], [6], [8].

Suppose $K$ be a nonempty subset of a metric space $(X, d)$. A mapping $T: K \rightarrow K$ is said to be contractive if there exists $0 \leq \alpha<1$ such that for all $x, y \in X$,

$$
d(T x, T y) \leq \alpha d(x, y) .
$$

The constant $\alpha$ is called the contractivity coefficient. It is well known that Banach's fixed point theorem asserts that if $K=X, T$ is contractive and $(X, d)$ is complete, then $T$ has a unique fixed point $p$ in $X$, and for any $x_{0} \in X$ the sequence $\left\{T^{n}\left(x_{0}\right)\right\}$ converges to $p$. This result has been extended by several authors to some classes of mappings by changing the contractive condition (6). It is well known that the Banach contraction principle [1] is a very useful and classical tool in nonlinear analysis.

Our purpose in this paper to found common fixed point theorem by using a more general contractive condition and three-step iteration scheme (4) and employ the following cotractive condition: 
Let $K$ be a nonempty closed convex subset of a Banach space $X$ and $T$ : $K \rightarrow K$ be a self-mapping of $K$ such that for each $x, y \in X$ and $k_{n} \in[0,1)$, we define the following contractive condition (say M-contraction):

$$
\|T x-T y\| \leq e^{M\|x-T x\|}\left(k_{n}\|x-y\|\right),
$$

where $M \geq 0$ and $e^{x}$ denotes the exponential function of $x \in X$.

Example. Let $X$ be the real line with the usual norm $\|\cdot\|$ and suppose $K=[0,1]$. Define $T: K \rightarrow K$ by

$$
T x=\frac{x+1}{2} \text { and } T y=\frac{3 y-1}{2}
$$

for all $x, y \in K$. Obviously $T$ is self-mapping with the common fixed point 1 for all $x, y \in K$. Now we check that our condition $(7)$ is true. If $x, y \in[0,1]$. Moreover, $\|T x-T y\| \leq e^{M\|x-T x\|}\left(k_{n}\|x-y\|\right)$ for all $x, y \in[0,1]$. In fact

$$
\begin{aligned}
\|T x-T y\| & =\left\|\frac{(x+1)}{2}-\frac{(3 y-1)}{2}\right\|=\| \frac{(x+1)-(3 y-1)}{2} \\
& =\left\|\frac{x-3 y+2}{2}\right\|,
\end{aligned}
$$

and

$$
\begin{aligned}
e^{M\|x-T x\|}\left(k_{n}\|x-y\|\right) & =e^{M\left\|x-\frac{\mathrm{x}+1}{2}\right\|}\left(k_{n}\|x-y\|\right) \\
& =e^{M\left\|\frac{\mathrm{x}-1}{2}\right\|}\left(k_{n}\|x-y\|\right)
\end{aligned}
$$

Clearly, if we choose $x=0, y=1$, then contractive condition (7) is satisfied because

$$
\|T x-T y\|=\left\|\frac{x-3 y+2}{2}\right\|=\left\|\frac{-1}{2}\right\|=\frac{1}{2},
$$

and for $L \geq 0$ and $k_{n} \in[0,1)$, we chose $M=0$ and $k_{n}=0.5$, then

$$
\begin{aligned}
e^{M\|x-T x\|}\left(k_{n}\|x-y\|\right) & =e^{M\left\|x-\frac{x+1}{2}\right\|}\left(k_{n}\|x-y\|\right)=e^{M\left\|\frac{x-1}{2}\right\|}\left(k_{n}\|x-y\|\right) \\
& =e^{M(1 / 2)}\left(k_{n}\|0-1\|\right)=e^{0(1 / 2)}\left(\frac{1}{2}(1)\right)=\frac{1}{2}
\end{aligned}
$$

Therefore $\|T x-T y\| \leq e^{M\|x-T x\|}\left(k_{n}\|x-y\|\right)$. Hence $T$ is a self mapping with common fixed and satisfying contractive condition (7).

In order to prove our result, we need the following lemma [2]: 
Lemma 1. Let $\left\{a_{n}\right\},\left\{b_{n}\right\}$ and $\left\{t_{n}\right\}$ be the sequences of nonnegative numbers satisfying:

$$
\begin{aligned}
a_{n+1} & \leq\left(1-\omega_{n}\right) a_{n}+b_{n}+t_{n} \text { for all } n \geq 0 \text { where }\left\{\omega_{n}\right\}_{n=0}^{\infty} \subset[0,1] . \\
\text { If } \sum_{n=0}^{\infty} \omega_{n} & =\infty, b_{n}=O\left(\omega_{n}\right) \text { and } \sum_{n=0}^{\infty} t_{n}<\infty, \text { then } \lim _{n \rightarrow \infty} a_{n}=0 .
\end{aligned}
$$

\section{Main Results}

In this section, we have proved strong convergence theorem and find approximate common fixed points of self-mapping $T$. In the consequence, $F$ denotes the set of common fixed point of the mapping $T$.

Theorem 2. Let $K$ be a nonempty closed convex subset of Banach space $X$ and $T: K \rightarrow K$ be a self-mapping of $K$ satisfying contractive condition (7) with $F(T) \neq \phi$, where $F(T)$ is the set of fixed points of $T: K \rightarrow K$ be continuous. Suppose $\left\{x_{n}\right\}$ be the sequence defined by iteration (4) for arbitrary $x_{0} \in K$, where $\left\{\alpha_{n}\right\},\left\{\beta_{n}\right\}$ and $\left\{\gamma_{n}\right\}$ are sequences in $[0,1]$ with $\alpha_{n}+\beta_{n}+\gamma_{n}=1$ such that $\sum_{n=0}^{\infty} \alpha_{n}=\infty$. Then $\left\{x_{n}\right\}$ converges strongly to a common fixed point of $T$.

Proof. Suppose $F(T) \neq \phi$ and $p \in F(T)$. Since $T$ is satisfying the contractive condition (7). For any $x, y \in K$. Suppose $\mathrm{p}$ is a fixed point of $T$ now, put $x=p$ and $y=z_{n}$ in (7), we obtain

$$
\left\|T z_{n}-p\right\| \leq e^{M\|p-T p\|}\left(k_{n}\left\|z_{n}-p\right\|\right)
$$

Suppose $\left\{x_{n}\right\}_{n=0}^{\infty}$ be the sequences defined by (4). Then we have

$$
\begin{aligned}
\left\|x_{n+1}-p\right\| & =\left\|\left(1-\alpha_{n}\right) z_{n}+\alpha_{n} T z_{n}-p\right\| \\
& =\left\|\left(1-\alpha_{n}\right)\left(z_{n}-p\right)+\alpha_{n}\left(T z_{n}-p\right)\right\| \\
& \leq\left(1-\alpha_{n}\right)\left\|z_{n}-p\right\|+\alpha_{n}\left\|T z_{n}-p\right\| .
\end{aligned}
$$

From (8) and (9), we get

$$
\begin{aligned}
\left\|x_{n+1}-p\right\| & \leq\left(1-\alpha_{n}\right)\left\|z_{n}-p\right\|+\alpha_{n} e^{M\|p-T p\|}\left(k_{n}\left\|z_{n}-p\right\|\right) \\
& \leq\left(1-\alpha_{n}\right)\left\|z_{n}-p\right\|+\alpha_{n} e^{M\|p-p\|}\left(k_{n}\left\|z_{n}-p\right\|\right) \\
& \leq\left(1-\alpha_{n}\right)\left\|z_{n}-p\right\|+\alpha_{n} e^{M(0)}\left(k_{n}\left\|z_{n}-p\right\|\right) \\
& \leq\left(1-\alpha_{n}\right)\left\|z_{n}-p\right\|+\alpha_{n} k_{n}\left\|z_{n}-p\right\| \\
& \leq\left(1-\alpha_{n}+\alpha_{n} k_{n}\right)\left\|z_{n}-p\right\| .
\end{aligned}
$$


Taking $x=p$ and $y=y_{n}$ in (7), we obtain

$$
\left\|T y_{n}-p\right\| \leq e^{M\|p-T p\|}\left(k_{n}\left\|y_{n}-p\right\|\right)
$$

Now,

$$
\begin{aligned}
\left\|z_{n}-p\right\| & =\left\|\left(1-\gamma_{n}\right) y_{n}+\gamma_{n} T y_{n}-p\right\| \\
& =\left\|\left(1-\gamma_{n}\right)\left(y_{n}-p\right)+\gamma_{n}\left(T y_{n}-p\right)\right\| \\
& \leq\left(1-\gamma_{n}\right)\left\|y_{n}-p\right\|+\gamma_{n}\left\|T y_{n}-p\right\| .
\end{aligned}
$$

Now From (11) and (12), we obtain

$$
\begin{aligned}
\left\|z_{n}-p\right\| & \leq\left(1-\gamma_{n}\right)\left\|y_{n}-p\right\|+\gamma_{n} e^{M\|p-T p\|}\left(k_{n}\left\|y_{n}-p\right\|\right) \\
& \leq\left(1-\gamma_{n}\right)\left\|y_{n}-p\right\|+\gamma_{n} e^{M\|p-p\|}\left(k_{n}\left\|y_{n}-p\right\|\right) \\
& \leq\left(1-\gamma_{n}\right)\left\|y_{n}-p\right\|+\gamma_{n} e^{M(0)}\left(k_{n}\left\|y_{n}-p\right\|\right) \\
& \leq\left(1-\gamma_{n}\right)\left\|y_{n}-p\right\|+\gamma_{n} k_{n}\left\|y_{n}-p\right\| \\
& \leq\left(1-\gamma_{n}+\gamma_{n} k_{n}\right)\left\|y_{n}-p\right\| .
\end{aligned}
$$

Substitute (13) into (10) implies that

$$
\left\|x_{n+1}-p\right\| \leq\left(1-\alpha_{n}+\alpha_{n} k_{n}\right)\left(1-\gamma_{n}+\gamma_{n} k_{n}\right)\left\|y_{n}-p\right\| .
$$

Now, put $x=p$ and $y=x_{n}$ in (7), we obtain

$$
\left\|T x_{n}-p\right\| \leq e^{M\|p-T p\|}\left(k_{n}\left\|x_{n}-p\right\|\right)
$$

Now,

$$
\begin{aligned}
\left\|y_{n}-p\right\| & =\left\|\left(1-\beta_{n}\right) x_{n}+\beta_{n} T x_{n}-p\right\| \\
& =\left\|\left(1-\beta_{n}\right)\left(x_{n}-p\right)+\beta_{n}\left(T x_{n}-p\right)\right\| \\
& \leq\left(1-\beta_{n}\right)\left\|x_{n}-p\right\|+\beta_{n}\left\|T x_{n}-p\right\| .
\end{aligned}
$$

Now From (15) and (16), we get

$$
\begin{aligned}
\left\|y_{n}-p\right\| & \leq\left(1-\beta_{n}\right)\left\|x_{n}-p\right\|+\beta_{n} e^{M\|p-T p\|}\left(k_{n}\left\|x_{n}-p\right\|\right) \\
& \leq\left(1-\beta_{n}\right)\left\|x_{n}-p\right\|+\beta_{n} e^{M\|p-p\|}\left(k_{n}\left\|x_{n}-p\right\|\right) \\
& \leq\left(1-\beta_{n}\right)\left\|x_{n}-p\right\|+\beta_{n} e^{M(0)}\left(k_{n}\left\|x_{n}-p\right\|\right) \\
& \leq\left(1-\beta_{n}\right)\left\|x_{n}-p\right\|+\beta_{n} k_{n}\left\|x_{n}-p\right\| \\
& \leq\left(1-\beta_{n}+\beta_{n} k_{n}\right)\left\|x_{n}-p\right\| .
\end{aligned}
$$


Substitute (17) into (14), we get

$$
\begin{aligned}
\left\|x_{n+1}-p\right\| & \leq\left(1-\alpha_{n}+\alpha_{n} k_{n}\right)\left(1-\beta_{n}+\beta_{n} k_{n}\right)\left(1-\gamma_{n}+\gamma_{n} k_{n}\right)\left\|x_{n}-p\right\| \\
& \leq\left[1-\alpha_{n}\left(1-k_{n}\right)\right]\left[1-\beta_{n}\left(1-k_{n}\right)\right]\left[1-\gamma_{n}\left(1-k_{n}\right)\right]\left\|x_{n}-p\right\|
\end{aligned}
$$

i.e.,

$$
\left\|x_{n+1}-p\right\| \leq\left[1-\alpha_{n}\left(1-k_{n}\right)\right]\left\|x_{n}-p\right\| .
$$

Since since $0 \leq k_{n}<1, \alpha_{n} \in[0,1]$, and $\sum_{n=0}^{\infty} \alpha_{n}=\infty$ and setting $a_{n}=$ $\left\|x_{n}-p\right\|, \omega_{n}=\alpha_{n}\left(1-k_{n}\right)$ by lemma 1 , we obtain

$$
\lim _{n \rightarrow \infty}\left\|x_{n+1}-p\right\|=0 .
$$

So that $\left\{x_{n}\right\}_{n=0}^{\infty}$ converges strongly to the common fixed point of $\mathrm{T}$.

Proof of the Uniqueness. suppose $p_{1}, p_{2} \in F(T)$, where $F(T)$ is the set of fixed points of $T$ in $X$ such that $p_{1}=T p_{1}$ and $p_{2}=T p_{2}$.

Let on the contrary that $p_{1} \neq p_{2}$. Then, using contractive condition (7), we get

$$
\begin{aligned}
\left\|p_{1}-p_{2}\right\| & =\left\|T p_{1}-T p_{2}\right\|=e^{M\left\|p_{1}-T p_{1}\right\|}\left(k_{n}\left\|p_{1}-p_{2}\right\|\right) \\
& =e^{M\left\|p_{1}-p_{1}\right\|}\left(k_{n}\left\|p_{1}-p_{2}\right\|\right) \\
& =e^{M(0)}\left(k_{n}\left\|p_{1}-p_{2}\right\|\right) \\
& =k_{n}\left\|p_{1}-p_{2}\right\|<\left\|p_{1}-p_{2}\right\|
\end{aligned}
$$

which is a contradiction. Therefore, $p_{1}=p_{2}$.

This complete the proof.

The following results are immediate sequel of our strong convergence theorem.

Corollary 3. Let $K$ be a nonempty closed convex subset of Banach space $X$ and $T: K \rightarrow K$ be a self-mapping of $K$ satisfying contractive condition (7) with $F(T) \neq \phi$, where $F(T)$ is the set of fixed points of $T: K \rightarrow K$ be continuous. Suppose $\left\{x_{n}\right\}$ be the sequence defined by iteration (2) for arbitrary $x_{0} \in K$, where $\left\{\alpha_{n}\right\},\left\{\beta_{n}\right\}$ and $\left\{\gamma_{n}\right\}$ are sequences in $[0,1]$ with $\alpha_{n}+\beta_{n}+\gamma_{n}=1$ such that $\sum_{n=0}^{\infty} \alpha_{n}=\infty$. Then $\left\{x_{n}\right\}$ converges strongly to a common fixed point of $T$.

Corollary 4. Let $K$ be a nonempty closed convex subset of Banach space $X$ and $T: K \rightarrow K$ be a self-mapping of $K$ satisfying contractive condition (7) with $F(T) \neq \phi$, where $F(T)$ is the set of fixed points of $T: K \rightarrow K$ be continuous. Suppose $\left\{x_{n}\right\}$ be the sequence defined by iteration (3) for arbitrary 
$x_{0} \in K$, where $\left\{\alpha_{n}\right\},\left\{\beta_{n}\right\}$ and $\left\{\gamma_{n}\right\}$ are sequences in $[0,1]$ with $\alpha_{n}+\beta_{n}+\gamma_{n}=1$ such that $\sum_{n=0}^{\infty} \alpha_{n}=\infty$. Then $\left\{x_{n}\right\}$ converges strongly to a common fixed point of $T$.

When $\beta_{n}=0$ then given by Thianwan [11] iteration scheme is corollary of our result.

Corollary 5. Let $K$ be a nonempty closed convex subset of Banach space $X$ and $T: K \rightarrow K$ be a self-mapping of $K$ satisfying contractive condition (7) with $F(T) \neq \phi$, where $F(T)$ is the set of fixed points of $T: K \rightarrow K$ be continuous. Suppose $\left\{x_{n}\right\}$ be the sequence defined by iteration (5) for arbitrary $x_{0} \in K$, where $\left\{\alpha_{n}\right\},\left\{\beta_{n}\right\}$ and $\left\{\gamma_{n}\right\}$ are sequences in $[0,1]$ with $\alpha_{n}+\beta_{n}+\gamma_{n}=1$ such that $\sum_{n=0}^{\infty} \alpha_{n}=\infty$. Then $\left\{x_{n}\right\}$ converges strongly to a common fixed point of $T$.

\section{References}

[1] S. Banach, Sur les operations dans les ensembles abstraits et leur application aux equations integerales, Fund Math., 3 (1922), 133-181.

[2] V. Berinde, Iterative Approximation of Fixed Points, Springer-Verlag Berlin Heidelberg (2007).

[3] V. Berinde, On the convergence of the Ishikawa iteration in the calss of quasi-contractive operators, Act. Math. Univ. Comenianae, LXXXIII, No. 1 (2004), 1-11.

[4] V. Berinde, On the convergence theorem for Mann iteration in the calss of Zamfirescu operators, Analele Universitatii de Vest. Timisoara Seria Mathematica-Informatic, XLV, No. 1 (2007), 33-41.

[5] S. Ishikawa, Fixed points by a new iteration method, Proc. Amer. Math. Soc., 44 (1974), 147-150.

[6] O. Olaleru Johnson, On the convergence rates of Picard, Mann and Ishikawa iteration of generalized contractive operators, Studio Univ. "Bages-Balyat", Matheamtica, LIV, No. 4 (2009), 103-114.

[7] W.R. Mann, Mean value methods in iteration, Proc. Amer. Math. Sco., 4 (1953), 506-510. 
[8] S. Pulickakunnel, N. Sing, Approximation of common fixed points for a finite family of Zamfirescu operators, Gneral Math., 18, No. 4 (2010), 175184.

[9] B.E. Rhoades, Fixed point iteration using infinite matrices, Trans. Amer. Math. Soc., 196 (1974), 161-176.

[10] B.E. Rhoades, Comments on two fixed point iteration methods, J. Math. Anal. Appl., 56, No. 2 (1976), 741-750.

[11] S. Thianwan, Common fixed points of the new iterations for two asymptotically nonexpansive nonself-mappings in a Banach space, J. Comput Appl. Math., 224 (2009), 688-695.

[12] T. Zamfirescu, Fix point theorems in matric spaces, Arch. Math., 23 (1972), 292-298. 
